Aus der chirurgischen Universitätsklinik zu Königsberg i. Pr. (Direktor: Prof. Dr. E. Le x e r).

\title{
Über die Behandlung des typischen Radiusbruches.
}

\author{
Von Dr. Walter Krantz.
}

Úberblickt man die Zahl der für den typischen Radiusbruch angegebenen Behandlungsmethoden, so ist man erstaunt über die Fülle der verschiedenen Vorschläge, die zu dem erstrebten Ziele, der Wiederherstellung der natürlichen Form des Knochens und vor allem der Erhaltung der Gebrauchsfähigkeit, führen sollen. Wie so oft in der Medizin ist aber auch hier die Menge der vorgeschlagenen Methoden ein Zeichen des vergeblichen Suchens nach einer wirklich rationellen, ein weiterer Beweis aber auch für die Schwierigkeit des Gegenstandes. Hierzu kommt noch die Häufigkeit des Bruches. B ardenheuer betont, daß er in dieser Beziehung die erste Stelle unter den Knochenbrüchen einnimmt. Dupuytre $\mathrm{n}, \mathrm{H}$ offa und $\mathrm{S}$ tor $\mathrm{p}$ berechnen sein Vorkommen auf Io Proz. aller Brüche. B r u n s gar auf I8 Proz. Das weibliche Geschlecht scheint etwas bevorzugt. Nach einer Statistik von M o r r is entfallen von 169 typischen Radiusfrakturen II 4 auf Frauen, 55 auf Männer. Bei unseren Patienten waren unter 243 Fällen I $57_{7}$ Frauen und 86 Männer. Nach $\mathrm{H}$ of $\mathrm{fa}$ stellt das größte Kontingent das 50.-6o. Lebensjahr, dann das vierte und erste Dezennium, während das zweite und dritte nur wenig beteiligt sind. Auch von unserem Krankenmaterial entfallen die meisten Fälle auf das 50.-6o. Lebensjahr, es folgt dann das fünfte und siebente Dezennium, in ziemlich weitem Abstand das zweite bis vierte, die unter sich ziemlich gleiche Zahlen aufweisen, während dagegen das erste Dezennium nur sehr wenig betroffen ist.

Bevor wir die verschiedenen Behandlungsmethoden einer näheren Betrachtung unterziehen, wollen wir mit einigen Worten 
auf Wesen, Bedeutung, Entstehung und Folgen der typischen Radiusfraktur eingehen, deren Kenntnis ja zur Vornahme der Reposition und des Verbandes nicht unwesentlich ist.

„,Typisch“ bezieht sich hier auf den Sitz der Fraktur, weswegen man sie auch, wohl mit mehr Recht, Fraktura radii loco classico genannt hat. Die Bezeichnung ,typisch“ ist, obgleich sie nicht korrekt ist, der Kürze wegen und aus Gewohnheit von den älteren Autoren übernommen und hat sich heute allgemein eingebürgert.

Die Fraktur liegt etwa $\mathrm{I}-2 \mathrm{~cm}$ oberhalb des Handgelenks und fällt im jugendlichen Alter häufig mit einer Epiphysenlösung zusammen. - Recht verschieden waren die Meinungen lange Zeit über die Art des Bruches. Die einen nahmen einen queren Verlauf der Bruchlinie an, die anderen wieder meinten, daß dieselbe schräg verliefe. Die Mehrzahl der Autoren glaubte jedenfalls, daß reine Querbrüche nur selten seien. So wogte der Streit der Meinungen hin und her, bis das Röntgenverfahren die Entscheidung brachte und zeigte, daß beide Ansichten zu Recht bestanden, daß sowohl Quer- wie Schrägbrüche vorkommen und außerdem noch andere Formen, die man vorher nicht hatte nachweisen können. Gewöhnlich verläuft die Bruchlinie an einer Seite der Gelenkfläche näher als an der anderen, und zwar meist von distal volarwärts nach proximal dorsalwärts. Häufig geht sie in das Gelenk hinein, namentlich bei Schrägbrüchen, oder indem bei Querbrüchen das untere Fragment noch in vertikaler Richtung gesprengt ist.

Die gewöhnliche Dislokation ist die, daß das distale Fragment, am Proc. styloideus ulnae befestigt, eine Drehung um das untere Ulnaende macht, so daß es dorsalwärts verschoben wird. Es vollführt sozusagen eine Supination, während die Diaphyse eine Pronation erfährt. Die wichtigste Nebenverletzung ist die Absprengung des Proc. styloideus ulnae. $\mathrm{K}$ a h le y $B$ fand sie in 78 Proz. seiner Fälle, eine Zahl, die nach meinen Untersuchungen für unser Material viel zu hoch ist; sie beträgt bei uns 32 Proz.

Wir wenden uns nunmehr zur Beantwortung der Frage: Auf welche Weise und durch welchen Mechanismus entsteht die typische Radiusfraktur? Es ist doch zunächst auffallend, daß der Radius gerade an der Stelle, wo er am dicksten ist, so häufig bricht und nicht weiter oben in der Diaphyse. Eine große Anzahl, 
teilweise recht interessanter Theorien ist aufgestellt worden, diesen scheinbaren Widerspruch zu erklären. Schon lange bekannt war, daß der Bruch gewöhnlich durch indirekt einwirkende Gewalt und zwar durch Fall auf die pronierte und stark extendierte Hand erfolgt. P o n t e a u und L e P e tit verglichen nun Radius und Ulna mit Sehne und Bogen. Der Bogen wird bei seinem Fall über seine Elastizität zusammengebogen und bricht. Eine wenig durchdachte und wenig einleuchtende Erklärung, der denn auch bald andere folgten. Es war in der ersten Hälfte des vorigen Jahrhunderts hauptsächlich die Ansicht geltend, daß der typische Radiusbruch durch Coup und Contrecoup zustande komme. Dupuytren, Malgaigne und Nélaton waren die eifrigsten Verfechter dieser Idee. Letzterer suchte sie durch Leichenverusche $\mathrm{zu}$ beweisen und kommt zu folgender Erklärung: „Indem man bei einem Fall auf die ausgestreckte Hohlhand den Boden berührt, bildet der Vorderarm mit der Hand einen rechten Winkel; berücksichtigt man die Stellung der Carpalknochen hierbei, so ergibt sich, daß die erste Reihe derselben ein Gewölbe bildet, das sich auf den Boden stützt. Der fallende Körper drückt den Radius gegen dieses Gewölbe, und es müßte dasselbe entweder zerdrückt werden, oder es muß der Radius zerbrechen; da aber das Gewölbe fester ist, so tritt ein Bruch des Radius ein.“

Allmählich gewinnt dann eine Auffassung Boden, die lange Zeit allgemeine Geltung hatte und erst in der neuesten Zeit eine Einschränkung erfahren hat. B o u c h e t kam schon I834 zu der Überzeugung, daß der Radius nicht durch den Druck zweier entgegengesetzt wirkender Gewalten bräche, sondern dadurch, daß sein unteres Ende, der starken Extension der Hand folgend, gewissermaßen abgerissen werde. V o i 11 e m i e r erklärte das $\mathrm{Zu}$ standekommen von Querbrüchen durch den Zug des stark gespannten volaren Kapselbandes, welches das untere Fragment abreiße, während er für Schrägbrüche und eingekeilte Brüche eine direkte Gewalteinwirkung annahm. Auf diesen Ansichten weiter bauend, stellt dann L e c o m t e I860 den Satz auf, daß alle typischen Radiusbrüche ,par arrachement" entstehen. Er verwirft gänzlich die Lehre der Entstehung durch Coup und Contrecoup. Seine Theorie erlangte allgemeine Anerkennung und behielt sie lange Zeit hindurch, bis $1885 \mathrm{~L} \mathrm{ö} \mathrm{b} \mathrm{k} \mathrm{e} \mathrm{r} \mathrm{sie} \mathrm{mit} \mathrm{wohl-}$ 
durchdachten Gründen anfocht, die dann neun Jahre später von $\mathrm{B} \ddot{\mathrm{a} h \mathrm{r}}$ noch etwas modifiziert wurden. L ö bker faßt seine Untersuchungen dahin zusammen: ,Die Frakturen am unteren Drittel des Radius können mitunter ausschließlich durch direkten Stoß und Gegenstoß hervorgerufen werden; der typische Schrägbruch aber am unteren Ende des Knochens kommt durch eine kombinierte Wirkung beider genannten Kräfte zustande."

Eine interessante Erklärung gibt uns Hennequin. Er faßt die beiden Vorderarmknochen als zwei abgestumpfte Pyramiden auf, die in entgegengesetzter Richtung nebeneinander liegen. Das starke Lig. interosseum gleicht die Schwäche des unteren Radiusendes aus. Bei einem Fall auf die extendierte Hand wirkt auf den Radius der Widerstand der mit ihm fest verbundenen Karpalknochen und die über die Ulna durch das Lig. interosseum übertragene Gewalt des Humerus, der mit ihm selbst nur in losem Kontakt steht. Am unteren Ende, wo diese beiden Kräfte zusammentreffen, kommt es dadurch zu einem Einbruch des Radius. H e n n e qu i n läßt somit bei seiner Erklärung die Wirkung des Lig. carpi volare gänzlich außer Acht, er nähert sich der alten Theorie $\mathrm{M}$ a $1 \mathrm{~g}$ a i g $\mathrm{n}$ e s und $\mathrm{N}$ é $\mathrm{la}$ t o n s vom Coup und Contrecoup. B a r d e $\mathrm{n} \mathrm{he} \mathrm{u}$ e $\mathrm{r}$ ist der Ansicht, daß das distale Fragment durch das Lig. carpi volare resp. dorsale abgerissen werde. Helf e $\mathrm{r}$ c h bezeichnet die typische Radiusfraktur als kombinierte Riß- und Abknickungsfraktur, entstanden durch Zug des übermäßig gespannten Lig. carpi volare propr. und durch Anstemmen der oberen Karpalreihe an den dorsalen Vorsprung des unteren Radiusendes; er gibt damit die wohl heute am meisten verbreitete Ansicht wieder. Die typische Dislokation beim Radiusbruch wird teils auf die Gewalteinwirkung selbst, teils auf Muskelzug zurückgeführt. Helf e rich erklärt sie dadurch, daß das Gewicht des fallenden Körpers weiterwirkt und den Radiusschaft an dem distalen Fragment vorbeitreibt, bis er den Boden erreicht. H of $\mathrm{f}$ a geht weiter, indem er annimmt, daß die Dislokation zwar durch die Gewalteinwirkung hervorgerufen wird, aber durch Muskelzug erhalten wird. Er folgt darin den Ansichten von Colles, S m ith und Luc a s und führt einen interessanten Beweis von $\mathrm{Luc}$ a s an: Eine Frau mit typischem Radiusbruch starb. Eine Dislokation bestand nicht wegen Läh- 
mung der gesamten Armmuskulatur. Erst als die Totenstarre diese Muskeln ergriff, erfolgte die typische Dislokation. Viel seltener ist die Entstehung der typischen Radiusfraktur durch direkte Gewalt, z. B. durch Schuß, Uberfahren, Maschinenverletzung oder die zwar auch indirekte Entstehung durch Fall auf den Handrücken bei stark flektierter Hand. Es hat dann das Lig. carpi dorsale die Wirkung, die sonst das Lig. carpi volare ausübt. Demgemäß ist auch die Dislokation eine von der üblichen abweichende, das distale Fragment ist in solchen Fällen volarwärts und das proximale dorsalwärts verschoben.

Die Bedeutung der typischen Radiusfraktur, die man ohne weiteres schon aus dem großen Interesse, das ihr die Autoren entgegengebracht haben, schließen kann, liegt neben ihrer Häufigkeit auch in den Nebenverletzungen und Folgen, die sich an sie anschließen. Schon die typische Dislokation ist nicht immer leicht auszugleichen; ist die Reposition nicht vollständig, so ist eine Verkürzung des Radius und infolgedessen eine schlechte Stellung der Hand, gewöhnlich verbunden mit einer Funktionsstörung, die unausbleibliche Folge. Von schlimmer Bedeutung ist auch der Abriß des Proc. styloideus ulnae, desgleichen Splitterungen des Radius. Unschöne Knochenvorsprünge und schlechte Funktion sind das häufige Resultat, namentlich bei ungeeigneter Behandlung. Ein Verlauf der Bruchlinie in das Gelenk hinein zieht ebenfalls oft Störungen der Beweglichkeit nach sich. Luxationen der Ulna, Verletzungen der Gelenkkapsel oder einzelner Bänder sind ebenso verhängnisvoll wie schwer heilbar mit guter Funktion. Eine Anzahl von Komplikationen bleibt dann noch, die nur einer operativen Behandlung mit Aussicht auf Erfolg zugänglich sind: Verletzungen von Gefäßen, Nerven, Einkeilungen von Weichteilen zwischen die Knochen usw.

Ich wende mich nunmehr der Hauptaufgabe der vorliegenden Arbeit zu: einer kritischen Betrachtung der verschiedenen für den typischen Radiusbruch angegebenen Behandlungsmethoden. Wir erkennen in ihnen die mit den Zeiten wechselnden Grundzüge der Frakturbehandlung überhaupt wieder. Bei den älteren Autoren war die Wiederherstellung der natürlichen Form des Gliedes die erste Forderung, der man gerecht $\mathrm{zu}$ werden suchte durch Einrichten des Bruches und Erhalten in dieser Stellung durch 
Schienen und andere feststellende Verbände, die man so lange liegen ließ, bis die feste Vereinigung der Fragmente erfolgt war. Daß unter solchen Umständen die Erhaltung der normalen Funktion des Gliedes leiden mußte, ist selbstverständlich. Bei der langen Ruhe, $\mathrm{zu}$ der Muskeln und Gelenke verurteilt waren, büßten sie ihre Spannkraft ein, die Muskeln verfielen einer Inaktivitätsatrophie, und mühevolle, langdauernde Massage und Übungen waren nötig, den Schaden einigermaßen wieder gut $\mathrm{zu}$ machen. Leider ist dieses Prinzip der starren Verbände auch heute noch vielfach geltend, obgleich die Erfolge mit unserer modernen Behandlungsweise weit bessere geworden sind. Diese verfolgt als obersten Grundsatz: Erhaltung der völligen Gebrauchsfähigkeit des Gliedes. Die Wiederherstellung der natürlichen Form des gebrochenen Knochens wird daneben natürlich nicht vernachlässigt, sondern in gleicher Weise erstrebt, ist doch von ihr vielfach die Funktion abhängig.

Die ersten Behandlungsvorschläge für die typische Radiusfraktur haben heute hauptsächlich nur historisches Interesse, sind aber z. T. recht beachtenswert dadurch, daß wir vielfach Gedanken finden, die in neuerer Zcit wieder aufgetaucht und zu Geltung gekommen sind. Um dic Mitte des vorigen Jahrhunderts sind es komplizierte Schienenverbände von Goy rand, B landin, Stroh meyer, Schindler u. a., die mitcinander in Wettbewerb treten. Die Hand wurde hauptsächlich in ulnarer Abduktion erhalten. Großes Gewicht legte man auf die Erhaltung der normalen Breite des Zwischenknochenraumes, weil man fürchtete, daß durch eine Kallusverwachsung der Vorderarmknochen die Pronation und Supination leiden könnte. Man verwandte dazu cinen Schienenverband mit graduierten Kompressen, indem man einen schmalen Leinwandstreifen auf den Zwischenknochenraum legte, darüber immer breitere Streifen, so daß ein Keil entstand, der durch Bindentouren angewickelt wurde. Die Behandlungsdauer war recht beträchtlich. „Am 20. bis 30. Tage kann man den Verband entfernen, um Steifheit des Handgelenks zu verhüten", heißt es in Burgers Verbandlehre.

M a lg a i g n e stellt drei Forderungen auf bei Brüchen mit Dislokation: I. Wiederherstellung des Zwischenknochenraumes, 
2. Verbesserung der Dislokation des unteren Fragments nach hinten oder nach vorn, 3. Berichtigung der Abduktion der Hand, indem der Proc. styloideus auf sein natürliches Niveau zurückgeführt wird. Letztere sucht $\mathrm{Cl}$ i n e zum erstenmal zu erfüllen durch einen Vorschlag, der eine gewisse Ähnlichkeit mit ganz modernen Verfahren hat. Der Arm wurde zwischen zwei Schienen gefaßt und in eine Schlinge gelegt derart, daß die Hand durch ihr eigenes Gewicht nach der ulnaren Seite herüberhing. D u p u y$\mathrm{t} \mathbf{r}$ e $\mathrm{n}$ bediente sich zu diesem $Z$ weck einer gekrümmten Ulnarschiene. M a lgaig n e berichtet jedoch gleichzeitig, daß gute Erfolge bei diesem und ähnlichen Verfahren nicht zu verzeichnen waren, so daß eine andere Idee der Behandlung auftauchte, die ebenfalls heute - allerdings wesentlich modifiziert und verbessert - eine bedeutende Rolle spielt: die permanente Extension. Godin und Diday sind ihre Urheber. Näheres über ihre Methode ist leider nicht erhalten geblieben. Ihre Gedanken nahmen $\mathrm{Hugui}$ er und $\mathrm{Vel}$ pea $\mathrm{u}$ auf. Beide konstruierten äußerst umständliche Schienen, die dazu noch für die Kranken derartig beschwerlich und schmerzhaft waren, daß man von ihrer weiteren Verwendung bald Abstand nehmen mußte. Beachtenswert ist der schon von $\mathrm{M} \mathrm{al} \mathrm{g}$ a i g $\mathrm{n}$ e aufgestellte, auch heute unverändert bestehende Grundsatz, die Schienen nur bis zur ersten Reihe der Handwurzelknochen reichen zu lassen, um ein Steifbleiben der Finger zu verhüten.

Eine Modifikation des $\mathrm{C} l$ in e schen Verfahrens berichtete $\mathrm{C}$ e $\mathrm{B}$ ner. Man kann die Stellung der Hand gegen den Ulnarrand am einfachsten dadurch erreichen, daß man die Hand über eine Tragbinde herabhängen läßt; durch ihre eigene Schwere wird sie dann gegen den Ulnarrand gerichtet. $\mathrm{R}$ o be r $\mathrm{t}$ läßt, wie gleichfalls $C$ e $B \mathrm{ner}$ mitteilt, den Patienten acht Tage $\mathrm{zu}$ Bett liegen, während die Hand über ein Spreukissen herabhängt. Wir finden dann andererseits wieder Anschauungen, namentlich von seiten der Engländer und Amerikaner, die eine Biegung der Hand nach der ulnaren Seite für unzweckmäßig halten und infolgedessen einfache gerade Dorsal- oder Volarschienen anwenden. Colles, Barton und Skey vertreten um die Mitte des vorigen Jahrhunderts diesen Standpunkt, und letzterer bemerkt: „Große Sorgfalt ist nötig, das Fallen der Hand zu hindern, und 
dies wird erreicht, wenn man den ganzen Arm und die Hand in eine gut angelegte Schlinge einschließt."

Fanger von Copenhagen hat folgenden Versuch unternommen. Er legt Vorderarm und Hand über eine doppelt geneigte Ebene. Der Vorderarm steht in Pronation - der erste Hinweis auf dieses durchaus notwendige Repositionsmanöver. Die Hand hängt frei über das Ende der Ebene herunter. V e l$\mathrm{p}$ e a $\mathrm{u}$ ist auf Grund von Versuchen mit dieser Methode in der Charité nicht zufrieden mit ihr, bemerkt aber, daß diese Versuche nicht als endgültig beweisend angesehen werden könnten. Andere Schienen, die auch heute noch in Benutzung sind, sollen erst später ihre Besprechung finden.

Jahrzehntelang war dann hauptsächlich der zirkuläre Gipsverband im Gebrauch, der auch heute noch trotz besserer Methoden vielfach angewandt wird. Ihm vorauszugehen hat natürlich die Reposition der Fragmente, die derart vorgenommen wird, daß man die Hand extendiert, eine ulnare Abduktion und schließlich eine Volarflexion macht. Man gleicht damit nacheinander die Dislocatio ad longitudinem, ad latus und ad axin aus. Der Arm soll in der Mittelstellung zwischen Pronation und Supination stehen. $\mathrm{Zu}$ beachten ist ferner noch die vorhin erwähnte Forderung $\mathrm{M}$ a $\mathrm{lg}$ a i g n es, die Finger frei zu lassen. Schon beim Anlegen des Verbandes begegnet man großen Schwierigkeiten, die Fragmente in der richtigen Reposition zu erhalten, da man nach einigen Bindentouren gar keine Kontrolle mehr über das Glied hat. Es herrscht vielfach die Anschauung, daß die Fragmente wenig Neigung hätten, sich wieder in der alten Weise zu dislozieren, so daß es ein Leichtes sei, die gewonnene Reposition bis zum Erhärten des Verbandes zu erhalten. Mit vollem Recht tritt B a $r$ $\mathrm{d}$ e $\mathrm{n}$ h e u e $\mathrm{r}$ dieser Ansicht entgegen. Daher empfiehlt er auch, frühzeitig den Gipsverband zu erneuern, um sich von der richtigen Stellung der Fragmente zu überzeugen und sie womöglich zu verbessern. Leider wird diese Mahnung in der Praxis häufig nicht befolgt.

Als weiterer Nachteil gesellt sich die Gefahr der ischämischen Muskelentzündung hinzu, die namentlich beim Anlegen durch eine ungeübte Hand sich leicht einstellen kann, falls nicht andere Ursachen, wie Arterienzerreißung, Nervenquetschung, Thrombose 
usw. vorliegen. Ferner begegnet man häufig Druckgeschwüren, Phlegmonen und gar Nekrose durch nicht sachgemäßes Anlegen des Gipsverbandes, Gefahren, denen man seine Patienten nicht aussetzen sollte. Neuerdings ist von $\mathrm{M}$ ü $11 \mathrm{er}$ aus der Charité über günstige Resultate mit einem dort angewandten leichten Gipsverbande berichtet, worauf ich noch später zurückkomme.

In Erkenntnis der Nachteile des Gipsverbandes nahm man dann seine Zuflucht wiederum zu Schienen. Die Grundzüge der Schienenbehandlung sind die nämlichen, wie man sie früher hatte. Wir finden Schienen mit Flexion, solche mit ulnarer Abduktion und andere, die beide Biegungen gleichzeitig aufweisen. Vielfach empfohlen ist die Schedesche Volarschiene. Sie hat eine starke Flexion und eine leichte ulnare Abknickung. Durch Bindentouren wird sie an dem gebrochenen Gliede befestigt. Die C a r r sche Schiene trägt an ihrem unteren Ende ein konvexes hölzernes Lager, das der Radiusform entsprechend auf der radialen Seite höher ist als auf der ulnaren und am Handgelenk ganz niedrig wird. Außerdem befindet sich am Ende der Schiene ein rundliches Querstück, das von der Hand umfaßt wird, wodurch eine leichte Flexion ausgeübt wird. $\mathrm{C}$ a r r verzichtet auf eine ulnare Abknickung. $\mathrm{Köllicker}$ hat eine andere Schiene empfohlen, die auch wieder eine Flexion und ulnare Abduktion bezweckt. $\mathrm{N}$ élatons Pistolenschiene wirkt in ähnlicher Weise wie die $\mathrm{S}$ chedesche, nur wird sie dorsal angelegt. Eine ganz andere Stellung nimmt die Rosersche Dorsalschiene ein. R oser verbindet in Supinationsstellung, nachdem die gut gepolsterte Schiene, vom Condylus externus bis auf die Finger reichend, derart angelegt ist, daß sie nur bis zum Handgelenk dem Arm anliegt, während der von da ab freibleibende Raum durch keilförmige Leinwandkompressen ausgefüllt wird. Durch diesen Keil wird das untere Fragment volarwärts gedrängt, während das obere durch Bindentouren an die dorsale Schiene gezogen wird. H e lf e r i c h empfiehlt die Schiene für schwierige Fälle, erwähnt aber gleichzeitig, daß sie für den allgemeinen Gebrauch zu umständlich und voluminös ist. Neuere Vorschläge zielen dahin, die Schienen für jeden Fall gewissermaßen neu herzustellen. Es hat dies den Vorteil, daß sie besser für das Glied passen als die starren, für alle Fälle gegebenen Holz- oder Eisenschienen. Unter 
diesen Vorschlägen möchte ich nur die von B e e 1 y und B r a a t $\mathrm{z}$ erwähnen, da andere eine nennenswerte Verbreitung nicht gefunden haben. B e ely fertigt seine Schiene aus Gips und Hanf. Die Hand wird flektiert, ein wenig ulnarwärts abduziert, während ein Gegenzug am Oberarm ausgeübt wird. Die Schiene wird nun radial und dorsal angelegt und durch einige Bindentouren angewickelt, worauf der Arm in eine bis zum Handgelenk reichende Mitella kommt.

Eine Kombination einer volaren und dorsalen Schiene und Modifikation der Beely schen stellt die Bra t z'sche Gipszeugschiene dar. Man benötigt zur Herstellung eines sackleinwandähnlichen Gewebes, Hessian. Dieses wird in der erforderlichen Weise zurechtgeschnitten und mit einem Gipsbrei durchtränkt. Die so präparierte Schiene wird an den redressierten Arm angelegt und bis zum Erstarren in der gewünschten Repositionsstellung erhalten. Über das ganze kommen Bindentouren, worauf der Arm in eine Mitella gelegt wird. Die B r a a t z'sche Schiene hat vor anderen, mit Ausnahme der von B e ely angegebenen, den Vorzug der individuellen Anpassung an die jeweiligen Konturen des gebrochenen Gliedes, wodurch ein festeres Anliegen des Verbandes und ein besseres Erhalten der Reposition gewährleistet wird.

Wie schon angedeutet, hat man sich jedoch in neuester Zeit von der Behandlung des typischen Radiusbruches durch feststellende Verbände abgewandt. B a r d e $n$ h e u e r beleuchtet die verschiedenen Schäden, die bei Anwendung von Schienen auftreten und warnt eindringlich vor ihnen. Sie teilen verschiedene Nachteile mit dem Gipsverband. Man muß sie, um ihre Wirkung nicht illusorisch zu machen, fest anlegen, wodurch die große Gefahr einer Druckphlegmone entsteht. Gelenk- und Sehnenscheidenentzündungen sind ebenfalls nicht selten. Durch die lange Ruhigstellung des Gliedes und die damit verknüpfte Retraktion der Gelenkkapsel und -bänder bildet sich häufig eine Ankylosis heraus, deren Zustandekommen durch eine übermäßige Kallusproduktion noch unterstützt wird. B a rde $\mathrm{nheuer}$ spricht die Überzeugung aus, daß bei festen Verbänden die Bildung von Kallus eine ungleich üppigere ist, als bei anderen, speziell seiner noch näher zu erörternden Extensionsbehandlung. Schließlich erwähnt er 
noch als Nachteil der Schienenbehandlung die lange Dauer derselben, die wiederum leicht zu Ankylosis und Atrophien führt. Als weiteren Nachteil möchte ich nochmals den Mangel einer individuellen Anpassung, den $\mathrm{B}$ e e $1 \mathrm{y}$ und $\mathrm{Br}$ a a $\mathrm{t} \mathrm{z}$ vermieden haben, hervorheben, wodurch die Dislokation sich häufig unter dem Verbande neu bildet, namentlich wenn die anderen, oben angeführten Momente: Abnahme der Bruchgeschwulst, des Fettpolsters usw. hinzukommen. Schließlich vermißt man bei allen in Gebrauch befindlichen starren Schienen die zur gehörigen Reposition erforderlichen Krümmungen. Es ist ja natürlich, daß man ein starres Gebilde, wie es die Schiene nun einmal ist, nicht derart formen kann, wie es zum vollkommenen Redressement der typischen Radiusfraktur notwendig ist. $\mathrm{Bra}$ a $\mathrm{t} z$ suchte dieser Indikation zu genügen durch seine formbare Gipszeugschiene; wir möchten daher auch ihr unter den Schienenverbänden den Vorzug geben. Immerhin besitzen wir heute Verfahren, die diese Forderung noch vollkommener erfüllen und auch sonst mancherlei Vorzüge haben. Den Schienenverbänden am nächsten steht das B a rde $\mathrm{nh}$ e u e r sche Extensionsverfahren, das uns bei so vielen Frakturen ausgezeichnete Dienste leistet. Zur ambulanten Behandlung der typischen Radiusfraktur hat B a r d e n$\mathrm{h}$ e u e $\mathrm{r}$ eine Federextensionsschiene konstruiert, die den Patienten nicht an das Bett fesselt. Die Schiene ist derart eingerichtet, daß eine an der volaren Seite liegende Brücke das obere Fragment dorsalwärts drückt. Durch einen Querzug wird das Handgelenk mit dem unteren Fragment radialwärts geleitet, während durch einen am Ende angebrachten Federzug die pronierte Hand volarwärts und ulnarwärts gelenkt wird. Durch zwei am Vorderarm angebrachte Gurte wird dieser fixiert. So sind in äußerst sinnreicehr Weise alle erforderlichen Repositionsmanöver bei Anlegung der Schiene gegeben. Als besonders wichtig ist $\mathrm{zu}$ bemerken, daB $\mathrm{B}$ a r d e $\mathrm{n}$ h e u e $\mathrm{r}$ auch auf die Pronation Wert legt; denn diese ist in der Tat durchaus notwendig, da ja beim Entstehen des Bruches der Schaftteil des Knochens in Pronationsstellung zu dem unteren Fragment kommt und darin verharrt. Die Anwendung der Schiene beträgt I2-I4 Tage, vom fünften Tage ab werden Bewegungsübungen im Handgelenk, namentlich Übung des Faustschlusses vorgenommen. Während der Übungen wird der Längs- 
zug und der radialwärts wirkende Querzug ausgehängt. Nach Abnahme der Schiene kommen Stabübungen an die Reihe. Nach drei bis vier Wochen soll der Patient gewöhnlich seine Tätigkeit wieder aufnehmen.

Bardenheuer hat eine große Anzahl von typischen Radiusbrüchen mit ausgezeichnetem Erfolge behandelt. Und das ist wohl ohne weiteres anzunehmen, denn sowohl die richtige Reposition der Fragmente, als die Erhaltung in derselben, die bei den bisher besprochenen Methoden so großen Schwierigkeiten begegnete, ist vollkommen geglückt, und wir können das Verfahren in funktioneller Hinsicht somit durchaus empfehlen, wenn es nicht einen Nachteil hätte, dem B a r d e $\mathrm{n} h$ e u e r selbst in seinem Vorwort schon begegnet: den der Umständlichkeit und des hohen Preises der Schienen. Eine Schiene für einen Erwachsenen kostet ca. Io5 M., die gleiche Auslage ist für eine kindliche Schiene erforderlich. Das ist doch für einen praktischen Artz eine recht erhebliche Ausgabe. GewiB hat B a r d e $\mathrm{n} h$ e u e r recht, wenn er sagt, daß selbst die größeren Kosten keine Rolle spielen dürften wegen der damit verbundenen bedeutend besseren funktionellen Resultate. Wir besitzen jedoch seit vier Jahren ein Verfahren, das funktionell vielleicht eben die guten Erfolge gibt, dessen Kosten dagegen gleich Null zu setzen sind. Andere Vorzüge werden bei der näheren Besprechung ihre Erwähnung finden. Es ist dies das Verfahren der beschränkten Feststellung mit Hilfe von Flanellbinden, von L e x e r auf dem Chirurgenkongreß I906 angegeben.

Zuvor möchte ich jedoch eine Methode besprechen, die gewissermaßen eine Reaktion auf die Schienenbehandlung darstellt und infolgedessen, wie so häufig, in ein Extrem verfällt, gegen das von vornherein Bedenken auftauchen müssen. Ich meine die Behandlung mittels Tragtuch von P e ters e n oder Suspensionsmanschette nach Stor p. Wie aus der Literatur ersichtlich, ist der Gedanke $\mathrm{P}$ e $\mathrm{t}$ e $\mathrm{r}$ s e $\mathrm{n}$ s nicht neu, sondern hat seine Vorläufer in $\mathrm{C} l$ in e, $\mathrm{C}$ e $\mathrm{B}$ ne r und $\mathrm{R}$ obert. Petersen legt den Arm nach vollzogener Reposition nur in eine Mitella, die bis zur Bruchstelle reicht, so daß von da ab die Hand frei in ulnarvolarer Flexionsstellung herabhängt. Es ist selbstverständlich, daß es dabei außerordentlich auf die Intelligenz und den guten Willen des 
Patienten ankommt, ja davon der ganze Erfolg abhängt. So beschränkt sich die Anwendung von vornherein auf eine kleine Zahl von Patienten. Von den meisten, namentlich dem poliklinischen Material, wird man die Einsicht und Willenskraft nicht verlangen können, trotz Schmerzen die Hand in der vorgeschriebenen Stellung zu erhalten. Wir müssen somit die Verallgemeinerung des P e t e r s e n schen Verfahrens verwerfen, mögen auch die Erfolge bei wirklich strikter Durchführung gute sein.

St o r p verwendet anstatt der bloßen Mitella einen etwa Io $\mathrm{cm}$ breiten Heftpflasterstreifen, der mehrfach um den Arm bis an den Proc. styloideus ulnae herangelegt wird. Durch einen darüber gelegten, mit einer Falte versehenen Streifen wird eine Binde gezogen, durch die der Arm am Nacken suspendiert wird. Eine gewisse Fixation und Kompression der Bruchstelle ist zwar, wie Storp als Vorteil hervorhebt, erreicht, doch ist dieselbe nicht so absolut, daß sie nicht durch unvernünftige Patienten willkürlich verändert werden könnte, einfach dadurch, daß sie den Daumen in die Weste oder Bluse stecken. Ferner muß man zur Vornahme von Massage den Verband jedesmal erneuern. Es ist zum mindesten umständlich, und das Abnehmen des Heftpflasterstreifens ist für den Patienten auch nicht angenehm. $S$ t o r p will demgemä $B$ in den ersten Io-I4 Tagen überhaupt nicht massieren; dies erscheint jedoch nach unseren Anschauungen wenig vorteilhaft für die spätere Funktion des Gliedes.

Es bleibt uns dann als das modernste Verfahren das der beschränkten Feststellung mit Hilfe von Flanellbinden, dessen Einführung wir $\mathrm{L}$ ex e $\mathrm{r}$ verdanken. Es erlaubt alle Bewegungen mit Ausnahme derjenigen, die zu einer Wiederverschiebung der Fragmente führen können. L e x e r suchte mit seinem Verfahren die Nachteile der Schienenbehandlung und der Behandlung ohne feststellenden Verband zu vermeiden, ihre Vorteile aber zu vereinen.

Wichtig zu einer tadellosen Heilung ist die genaue Ausführung der Reposition, die stets ohne Narkose, höchstens bei sehr empfindlichen Patienten in leichtem Ätherrausch vorgenommen wird. Ein Assistent umfaßt am rechtwinklig gebeugten Arm die Epikondylen, dann ergreift man mit einer Hand den Daumen, mit der anderen die vier Finger des verletzten Armes und bringt durch einen plötzlichen, kräftigen Ruck unter Ziehen am Daumen die 
Hand in Flexion, Pronation und ulnare Abduktion. L exe $\mathrm{r}$ legt, wie das stets auch v. B erg $\mathrm{m}$ a $\mathrm{n} n$ tat, einen großen Wert gerade auf die Pronation, deren Notwendigkeit sich ja aus der Entstehung des Bruches ergibt. Ferner ist wesentlich, einen kräftigen Zug auszuüben, wozu auch ein gut ausgeführter Gegenzug am Ellbogen gehört. Die Hand wird in der gewonnenen Repositionsstellung durch eine einfache Flanellbinde gehalten, die etwa eine Woche mit täglichem Wechsel getragen wird. Außerdem kommt der verletzte Arm in eine Mitella. Die Behandlungsdauer beträgt im ganzen zwei bis drei Wochen. Danach sind die meisten Patienten wieder arbeitsfähig. Beim Anlegen der Binde verfährt man nach L e x e r folgendermaßen: „Man beginnt über dem Epicondylus externus, führt sie von hier über den Handrücken und über den zweiten Mittelhandknochen (dessen Köpfchen bei mageren Händen etwas mit Watte gepolstert wird) hinweg; von da wird über die Vola und die ulnare Kante der Hand wiederum das Dorsum erreicht, sodann läuft die Binde vom radialen Rande des zweiten Metakarpus über die Vola zurück, gelangt oberhalb der Handgelenkgegend zur Streckseite des Vorderarms, um den sie in zwei Schlangentouren bis zum Epicondylus externus herumgeht. Von hier beginnen dieselben Gänge je nach Bedarf 2-3mal, wobei man die Hand noch stärker abduzieren, flektieren und pronieren kann, namentlich, wenn man beim zweiten Gange die Tour um die Hand herum ausläßt. Dies ist jedoch nur selten notwendig." Diese Art der Bindengänge ist von $\mathrm{L}$ exer seit I898 in der v. B e r g m a n n schen Poliklinik eingeführt und geübt worden; doch wurde stets noch eine entsprechend gebogene Pappschiene mit einer Organtinbinde darüber befestigt. Bei sehr empfindlichen Leuten bleibt der Verband 2-3 Tage liegen, sonst wird er gewöhnlich schon am zweiten Tage und von da ab täglich zur Vornahme von Massage und warmen Bädern abgenommen. Der Verband gestattet die freie Beweglichkeit der Finger und derjenigen Bewegungen im Handgelenk, die nicht zur Dislokation führen.

Das Verfahren stellte einen Versuch dar, auf einfachere Weise unter Vereinigung unserer Grundprinzipien der Frakturheilung und vielleicht sogar rascher als sonst zum Ziele zu kommen. Das jetzt vorliegende Material von 300 derartig behandelten Fällen 
ermöglicht es, über den Wert dieser Methode gegenüber anderen ein Urteil zu bilden, noch dazu, wo die Behandlung nicht von dem Autor des Verfahrens allein, sondern zum allergrößten Teile von Assistenten und jüngeren Hilfskräften der Klinik ausgeführt wurde. Der Gesamteindruck der erzielten Resultate war ein durchaus günstiger. Ich habe mich bemüht, möglichst viele der mit dem L e x e r - Verbande behandelten Patienten nachzuuntersuchen. Ein nicht kleiner Teil schied von vornherein aus, da er aus dem Auslande oder aus der Provinz und unerreichbar war. Ein anderer Teil war trotz Durchsicht der polizeilichen Bücher am Orte nicht mehr aufzufinden, so daß etwa 200 Patienten bestellt werden konnten, von denen IIo zu einer Nachuntersuchung erschienen. Es sind darunter Patienten jeden Lebensalters und der verschiedensten Berufe, so daß man sich auf Grund der gewonnenen Zahlen ein anschauliches Bild der erzielten Erfolge machen kann.

Ich habe bei der Nachuntersuchung die Stellung der Hand, die Supinationsfähigkeit, die Beugung und Hyperextension, sonstige Bewegungen wie Handrollen, Faustschluß usw. geprüft und durch Befragen des Patienten festgestellt, ob er seine Arbeitsfähigkeit wiedererlangt hat und ob noch irgendwelche Beschwerden von dem Bruch zurückgeblieben sind.

Sehen wir zunächst die Resultate in Bezug auf die Stellung der Hand an, so finden wir I5 Fälle, bei denen eine geringe radiale Verschiebung verzeichnet ist. Ein Fall ist dabei aber nicht in Betracht zu ziehen, weil bei ihm zugleich eine Klavikularfraktur bestand, die einen häufigen Verbandwechsel unmöglich machte. Bei einem anderen Fall, der sonst ausgezeichnet geheilt ist, handelt es sich um einen ins Gelenk reichenden Y-Bruch mit ziemlich erheblicher Splitterung. Ferner muß ich bemerken, daß in keinem

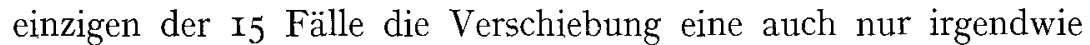
erhebliche ist. Tatsache ist, daß wir andererseits eine größere Anzahl von Fällen mit im Röntgenbilde nachweisbarer starker Dislokation haben, die ohne jede Verschiebung geheilt sind. Die radiale Verschiebung der Hand durch Verkürzung des Radius kommt bei ungenügender Extension namentlich dann vor, wenn ein Einkeilungsbruch vorliegt. Zwei Fehler der Behandlung sind dabei verantwortlich zu machen: I. Eine ungenügende Einrich- 
tung, 2. daß bei dem Verband durch einen Ungeübten die Hand mehr flektiert als ulnarwärts abduziert wird; es ist wenigstens möglich, daß hierdurch auf das untere Radiusende nicht der genügende $Z$ ug, vielleicht sogar ein gewisser Druck ausgeübt wird.

Wie schwierig es in manchen Fällen ist, die Fragmente richtig zu stellen, haben wir bei einer älteren Frau gesehen. Trotz mehrfacher sorgfältiger Repositionsmanöver gelang es nicht, die Hand richtig zu stellen. Auch ein Versuch in Narkose, desgleichen mit der R os e r'schen Supinationsstellung, mißlang. Da die Patientin leider nicht mehr zu erreichen war, war es uns nicht möglich, das schließliche Resultat kennen zu lernen.

Ich habe dann die Supination im einzelnen Falle näher geprüft und habe gefunden, daß sie in neun Fällen um ein ganz geringes zurückblieb, in vier Fällen bis $z u I 5^{\circ}$ und in sieben Fällen über $20^{\circ}$ gegenüber der gesunden Seite. In dem einen der letzten Fälle handelt es sich um einen Arbeiter, der seit seinem Radiusbruch eine Unfallrente bezieht. Derartige Patienten sind ja immer ein schwieriges Objekt für den Arzt und dürfen bei der Beurteilung eines Heilverfahrens nicht mit gleichem Maße gemessen werden. Ein anderer Fall, einen Faktor betreffend, bietet im übrigen ein recht erfreuliches Resultat, denn es bestand bei ihm ein Abriß des Proc. styl. ulnae neben ausgedehnter Splitterung des. Radius; das proximale Fragment war sehr stark nach unten disloziert. Der Patient ist wieder völlig arbeitsfähig. Die übrigen Fälle mit erheblicherer Supinationsbeschränkung betreffen durchweg alte Frauen zwischen 62 und 70 Jahren, die nicht ausschlieBlich auf ihrer Hände Arbeit angewiesen waren, somit die Nachbehandlung wohl nicht mit der nötigen Sorgfalt betrieben haben.

Bei der Prüfung der Flexion und Hypercxtension ergab sich, daß dieselbe bei 86 Patienten völlig oder doch nahezu normal war. Ich zähle hierzu auch die Fälle, die auch auf der gesunden Seite eine Behinderung in dieser Hinsicht zeigten, so daß der verletzte Arm nur unwesentlich davon abwich. Größere Abweichungen zeigten sich in 24 Fällen. Vier davon betrafen wiederum Frauen nahe den Siebzigern oder darüber. Ein anderer Fall wurde schon vorhin als besonders ungünstig erwähnt; es ist das der mit gleichzeitiger Klavikularfraktur. Zwei andere gingen mit ausgedehnterSplitterung des Radius, der eine davon mit Abriß des Proc. styloid., einher. 
Unter den übrigen Bewegungen habe ich hauptsächlich FaustschluB, seitliche Bewegungen und Handrollen geprüft. Dieselben waren in Io2 Fällen ausgezeichnet, in I Fall waren die seitlichen Bewegungen etwas beschränkt, in 7 Fällen war der Faustschluß ein nicht vollständiger, indem ein oder mehrere Finger der Hohlhand nicht völlig genähert werden konnten.

Schließlich handelte es sich um die Prüfung der Frage, die für den Patienten weitaus die wichtigste ist, um die Feststellung, ob der Patient nach der Behandlung wieder völlig arbeitsfähig ist. Da ergibt sich das erfreuliche Resultat, daß nur bei zwei Patienten angeblich eine Arbeitsunfähigkeit vorliegt, die man aber in beiden Fällen kaum der Behandlung zur Last legen kann. Der eine ist der schon mehrfach erwähnte Fall mit gleichzeitiger Klavikularfraktur, der infolgedessen nicht in der sonst üblichen Weise behandelt werden konnte und diesem Umstande wohl sein schlechtes Resultat verdankt. Beim zweiten Fall handelt es sich um einen Getreideträger, der objektiv einen recht günstigen Befund darbietet, der die Schmerzen, die angeblich bei ihm bestehen, nicht erklärt. Patient bezieht eine Unfallrente die wohl zu seiner ,Arbeitsbehinderung" beiträgt.

Bei 3 Patienten finden wir Angaben, daß sie ihre Arbeit nicht im alten Umfang versehen könnten. Zwei von ihnen beziehen gleichfalls Unfallrente; der eine bietet objektiv einen durchaus günstigen Befund dar; bei dem zweiten besteht eine geringe, radiale Verschiebung und eine Supinationsbeschränkung von $5^{\circ}$, während die übrigen Bewegungen nicht sonderlich behindert sind. Bei dem dritten dieser Patienten, der auch einen Antrag auf Rente gestellt hat, ist die Flexion und Hyperextension ziemlich stark behindert, auch gelingt der Faustschluß nicht vollständig. Der Bruch liegt vier Monate zurück; Patient kann augenblicklich noch nicht alles arbeiten.

In den übrigen I05 Fällen können die Patienten sämtlich ihre Arbeit in vollem Umfang wieder versehen. Ein Teil gibt auf Befragen leichte Beschwerden an: Schmerzen bei Witterungswechsel, zeitweise Schwäche im Arm nach größeren Anstrengungen usw. Nun, das sind Klagen, wie wir sie häufig nach Frakturen hören, die wohl bei jeder Form der Behandlung gelegentlich geäußert werden. 
Im Hinblick auf die späteren Störungen wäre es natürlich wünschenswert, über die Häufigkeit des abgerissenen Proc. styloideus bei den nachuntersuchten Fällen genaue Auskunft zu erhalten. Leider ist in dieser Beziehung das Material nicht geeignet, da aus pekuniären Gründen nicht von allen Patienten Röntgenaufnahmen angefertigt werden konnten. Nach den Röntgenbildern, die mir vorliegen, sind drei Fälle mit Abriß des Proc. styloideus ulnae. Von diesen interessiert uns die Gebrauchsfähigkeit ganz besonders. Bei dem einen Fall handelt es sich um eine 72 jährige Frau. Wir finden bei ihr weder eine Stellungsanomalie, noch eine Funktionsstörung; sie ist völlig beschwerdefrei. Allerdings hat die Behandlung bei ihr sechs Wochen gedauert. Der zweite Fall mit Abriß betrifft den schon oben näher erörterten Faktor. Er ist auch ohne Dislokation geheilt; doch besteht bei ihm eine Supinationsbeschränkung von $20^{0}$ und eine größere Störung in der Hyperextension. In seiner Arbeitsfähigkeit ist Patient jedoch nicht behindert. Bei einem 46 jährigen Schmied findet sich nur eine größere Beschränkung der Beugung und eine geringere der Überstreckung; im übrigen bietet er durchaus normale Verhältnisse. Als besonders günstig möchte ich noch hervorheben einen Fall, bei dem ein Y-Bruch ins Gelenk mit ziemlich starker Splitterung bestand, der mit Ausnahme einer geringen radialen Verschiebung ausgezeichnet geheilt ist. Auch bei einem anderen ebenfalls vollkommen gut ausgeheilten Fall bestand eine starke Splitterung. Bei zwei anderen, gleichfalls durch Röntgenbild kontrollierten Patienten ist der Bruch auch ohne jede anatomischen oder funktionellen Folgen gehcilt, obgleich eine recht starke Verschiebung des unteren Fragments bestand.

Die Zeit der wiedererlangten Gebrauchsfähigkeit läßt sich leider an dem gesamten Material nicht feststellen, zumal heutzutage sehr viele Patienten der Nachbehandlung durch die Tätigkeit des Kassenarztes entzogen werden. So können wir diese überaus wichtige Frage nur an einem Teile des Materials prüfen.

Wir haben uns dabei nicht auf die Angaben des Patienten verlassen, sondern nur auf Aufzeichnungen in den Büchern der Poliklinik, die namentlich in letzter Zeit ausgeführt sind. Danach haben wir mit Sicherheit festgestellt, daß bei mehreren Patienten die Zeit bis zur Wiedererlangung der Gebrauchsfähigkeit 
nur zwei Wochen betrug, bei sehr vielen war sie nach drei Wochen eingetreten, bei fast allen sicher am Ende der vierten Woche. Nur einige brauchten fünf und sechs Wochen, drei Patienten allerdings acht Wochen, einer zehn Wochen.

Unsere Resultate sind, in Prozentzahlen ausgedrückt:

13,6 Proz. radiale Verschiebung,

8,I Proz. geringe Supinationsbeschränkung über $5^{0}$;

Beugung und Úberstreckung ohne wesentliche Störungen bei 78,7 Proz.;

sonstige Bewegungen unbehindert in 92,7 Proz.;

die Arbeitsfähigkeit war vollkommen bei 94,7 Proz.;

beschränkt bei 2,7 Proz.;

völlig behindert bei 1,8 Proz.

In jüngster Zeit ist aus der chirurgischen Universitätspoliklinik der Königlichen Charité eine Dissertation von $\mathrm{M}$ ü ll e r erschienen, die genauere statistische Aufzeichnungen enthält über die Erfolge mit einem dort angewandten leichten zirkulären Gipsverband. M ülle $r$ hat Gelegenheit gehabt, neben den funktionellen Resultaten auch die anatomische Heilung durch Röntgenbilder $\mathrm{zu}$ kontrollieren. Wir konnten, wie schon bemerkt, dieselbe aus äußeren Gründen nicht überall durch das Röntgenbild kontrollieren, doch ist das an dem gut abzutastenden Gelenkende des Radius nur selten nötig. Da in der genannten Arbeit das L e x e r sche Verfahren häufig zum Vergleich herangezogen wird, seien auch hier einige Worte dazu gestattet. So möchte ich zunächst betonen, daß auch beim L exer-Verband die Möglichkeit zur Dislokation führender Bewegungen ausgeschlossen ist. Daß ein Patient zu Hause den Verband eigenmächtig entfernt hat, ist bei den über 300 derart behandelten Fällen nur einmal vorgekommen. Ich möchte meinen, daß dies auch bei einem leichten, nur mit einer Binde angelegten Gipsverbande möglich ist. Zuzugeben ist, daß eine Flanellbinde wohl etwas teurer ist als ein leichter Gipsverband; da sie jedoch nach Auswaschen mehrfach wieder benutzt werden kann, dürften sich die Kosten ungefähr ausgleichen.

M ü 11 e r sagt mit Recht, daß viele Wege zum Ziel führen. Es liegt auch uns fern, zu leugnen, daß mit anderen Behandlungsmethoden nicht vielleicht die gleichen Erfolge erzielt werden können, wie mit dem L ex e r-Verband. In einem Punkt aber 
ist er den meisten, namentlich den feststellenden, überlegen: in der Behandlungsdauer. Es sei mir gestattet, zum Vergleich die M üllersche Statistik heranzuziehen. Während dort unter 50 Fällen eine völlige Arbeitsfähigkeit nach zwei oder drei Wochen nur in sieben Fällen, dagegen nach zehn Wochen und darüber in acht Fällen eingetreten war, unter den übrigen etwa fünf bis sechs Wochen der Durchschnitt ist, waren bei uns, wie oben näher erörtert, nach spätestens vier Wochen fast alle Patienten wieder arbeitsfähig, nur einer nach zehn Wochen. Eine längere Zeit ist nach den Aufzeichnungen nicht festzustellen, wir erinnern uns auch einer solchen nicht.

Bei dieser Gelegenheit möchte ich einen Fehler richtigstellen. In einem Referat von $\mathrm{Ble} \mathrm{n} \mathrm{cke}$ in der Zeitschrift für orthopädische Chirurgie über die M ü 11 e r sche Dissertation findet sich die Bemerkung, L e x e r sei zum zirkulären Gipsverband zurückgekehrt. Ich bemerke ausdrücklich, daß dies keineswegs der Fall ist; uns ist unverständlich, wie Referent zu dieser Behauptung gekommen ist.

Ich möchte noch einigen Einwänden begegnen, die mir von einigen Seiten vorgehalten sind. Es wird gesagt, die Behandlung mit dem Lexer-Verbande sei sehr schmerzhaft. Von der Reposition muß man das wohlzugeben, doch bildet sie ja die Grundlage jeder Behandlungsmethode, ohne die wir nicht auskommen können. Unangenehm für den Patienten ist nur, daß derVerband jeden Tag wiederholt werden muß. Das Anlegen der Binde ist keineswegs schmerzhaft und wird von allen Patienten gut ertragen. Ferner wird behauptet, das Verfahren eigne sich nicht für die Praxis, weil man dazu zwei Assistenten brauche. Eine derartige Behauptung kann nur einer Unkenntnis des Verfahrens entspringen. Man kann, wenn man es nicht gerade mit Trunkenbolden $\mathrm{zu}$ tun hat, sehr gut die Reposition und das Anlegen der Binde völlig allein besorgen, wenn der Oberarm durch einen Bindenzügel am Fensterkreuz z. B. festgestellt wird. Bei Gips- oder Schienenverbänden dürfte es jedoch kaum möglich sein, ohne Hilfe die Hand beim Anlegen des Verbandes in Reposition zu erhalten. Auch hierin ist also das $\mathrm{L}$ e $\mathrm{x}$ e r sche Verfahren allen früheren Methoden überlegen und gewinnt gerade wegen seiner Einfachheit für den praktischen Arzt eine große Bedeutung. 
So darf ich nach meinen Untersuchungen behaupten, daß dieses Verfahren der beschränkten Feststellung sich bewährt hat, insofern als die Resultate mindestens ebensogut wie mit anderweitiger Behandlung sind, wenn nicht sogar bezüglich der Dauer der Wiederherstellung außerordentlich viel besser. Die Vorzüge des Verfahrens, kurz zusammengefaßt, sind: Einfachheit, Billigkeit, rasche Heilung, rasche Wiederherstellung der Beweglichkeit; die Nachteile, daß der Patient in den ersten Tagen bisweilen Beschwerden hat und täglich behandelt werden muß. Letzteres ist kein Nachteil für einen gewissenhaften Arzt, und was die Beschwerden in den ersten Tagen beim Massieren betrifft, so sind sie wohl kaum größer als bei den Patienten, bei welchen durch Verbände versteifte Gelenke durch kräftige passive Bewegungen wieder beweglich gemacht werden sollen. So kann ich das Verfahren auch für die Praxis durchaus empfehlen, aber es erfordert genaue Einhaltung der Vorschriften und eine richtige Einübung.

\section{Literaturverzeichnis.}

I. Frof. Dr. Bardenhe uer, Leitfaden der Behandlung von Frakturen und Luxationen I890, Ferd. Enke.

2. Prof. Dr. Barde $\mathrm{nh}$ e u er, Die Verletzungen der oberen Extremitäten. Deutsche Chirurgie 1888, Lief. 63b, Ferd. Enke.

3. Prof. Dr. Bardenheuer u. Dr. Rudolf Gräßner, Die Technik der Extensionsverbände bei der Behandlung von Frakturen und Luxationen der Extremitäten 1905, 2. Aufl., Ferd. Enke.

4. Dr. C. G. B u r g e r, Handbuch der chirurgischen Verbandslehre. Stuttgart, Riegersche Verlagsbuchhandlung 1849.

5. Dr. C. J. C e B n e r, Handbuch der chirurgischen Instrumenten- und Verbandslehre. 2. Aufl. Wien I 845 , Verlag von L. W. Seidel.

6. Maxim. Jos. Chelius, Handbuch der Chir., 6. Aufl. Wien, I843 Karl Gerold.

7. Dr. Paul Gla e $B$ ner, Zur Behandlung der typischen Radiusfraktur. Medizinische Klinik roog, Nr. 30.

8. F. H. H a m ilto n, Knochenbrüche und Verrenkungen. Nach der 5. Aufl. des englischen Originals unter Mitwirkung des Verfassers ins Dentsche übertragen von Dr, A. Rose. Göttingen I877, Vandenhoek \& Ruprecht, Verlag.

9. Prof. Dr. Helf e r i ch, Atlas und Grundriß der traumatischen Frakturen und Luxationen. 7. Aufl., j9o6. J. F. Lehmann-München.

Io. J. He $\mathrm{n} n$ equin, Considération sur le mécanisme, les symptomes et le traitement des fractures de l'extrémité inférieure du radius consécutives aux chutes sur le poignet. (Revue de Chirurgie 1894, Heft 7 u. 9.) Referat von Kramer im Zentralbl. f. Chirurgie I894. 
II. A 1 bert $\mathrm{H}$ of $\mathrm{f}$ a, Frakturen und Luxationen I904, 4. Aufl. Ferd. Enke.

I2. Dr. C. H u eter, Grundriß der Chirurgie. Leipzig i882, F. C. W. VogeJ.

I3. Dr. Max Kahl e y B, Beitrag zur Kenntnis der Frakturen am unteren Ende des Radius. Deutsche Zeitschr. f. Chirurgie Bd. 45.

I4 Dr. Franz König, Lehrbuch der speziellen Chirurgie Bd. 3, 5. Aufl. Berlin 1889 , August Hirschwald.

I 5. Prof. Dr. I. e x e r, Zur Behandlung der typischen Radiusfraktur. Verhandl. der Deutschen Gesellschaft f. Chirurgie I 906.

I6. Prof. Dr. Lexer, Zur Behandlung der Knochenbrüche. Münchn. med. Wochenschr. I909, Nr. I2.

17. J. F. M a $1 \mathrm{~g}$ a ig ne, Die Knochenbrüche und Verrenkungen. Deutsch von Dr. C. G. B u r g e r. Bd. I. Stuttgart I 850 . Riegersche Verlagsbuchhandlung.

18. Rein hold M ï $11 \mathrm{er}$. Statistischer Beitrag zur Behandlung des typischen Radiusbruches mittels zirkulärem Gipsverband. Inaug.-Dissert. Greifswald.

19. Jea nnot Stires, Über die Fraktura radii typica. Ein Beitrag zur Therapie. Inaug.-Diss. Königsberg.

20. Dr. Stor p, Zur Behandlung der typischen Radiusbrüche. Archiv f. klin. Chirurgie Bd. 53. Berlin 1896 , Aug. Hirschwald. 